\title{
Inhibitive effects of a novel efficient Schiff base on mild steel in hydrochloric acid environment
}

\author{
A.J.M. Eltmimi, ${ }^{1}$ A. Alamiery, ${ }^{2}$ (iD * A.J. Allami, ${ }^{3}$ R.M. Yusop, ${ }^{4}$ \\ A.H. Kadhum ${ }^{2,3}$ and T. Allami ${ }^{2}$ \\ ${ }^{1}$ Pharmacy Department AlRasheed University College, Baghdad, Iraq \\ ${ }^{2}$ Department of Chemical and Process Engineering, Faculty of Engineering and Built \\ Environment, Universiti Kebangsaan Malaysia, 43600 UKM Bangi, Selangor, Malaysia \\ ${ }^{3}$ University of Al-Ameed, Karbala, Iraq \\ ${ }^{4}$ Faculty of Science and Technology - FST, Universiti Kebangsaan Malaysia, 43000, \\ Bangi, Selangor, Malaysia \\ *E-mail: dr.ahmed1975@ukm.edu.my,dr.ahmed1975@gmail.com
}

\begin{abstract}
The corrosion inhibition performance of a novel Schiff base, namely 5-((4-fluorobenzylidene)amino)-1,3,4-thiadiazole-2-thiol (FATT), on mild steel in 1.0 M hydrochloric acid environment has been studied by the weight loss method and scanning electron microscope (SEM). The inhibition efficiencies and the adsorption characteristics were determined from the gravimetrical results. The inhibition effectiveness increases with an increase in the concentration of the studied inhibitor but decreases with rising solution temperature. The inhibitor molecules are adsorbed on the coupon (mild steel) surface and obey the Langmuir adsorption isotherm. Scanning electron spectroscopy (SEM) was utilized to test the coupon surface morphology without and with the inhibitor at a concentration of $0.5 \mathrm{mM}$ at $303 \mathrm{~K}$. SEM implies the formation of a protective layer of FATT molecules on the coupon surface in the hydrochloric acid solution at $303 \mathrm{~K}$. Quantum chemical calculations using density functional theory (DFT) were employed to identify the relationship between the electronic structure parameters of the FATT molecule and the inhibition efficiency. The quantum chemical parameters such as HOMO (highest occupied molecular orbital), LUMO (lowest unoccupied molecular orbital), $\Delta E$ (energy gap), $\mu$ (dipole moment), $\chi$ (electronegativity), $\eta$ (global chemical hardness), $\sigma$ (softness), and $\Delta N$ (fraction of electrons transferred) were calculated. The quantum chemical parameters by calculated the DFT method for the tested inhibitor agree with the experimental inhibition efficiency.
\end{abstract}

Keywords: fluorobenzylidene, mild steel, FATT, inhibitor, weight loss, scanning electron spectroscopy.

Received: May 30, 2020. Published: April 21, 2021

doi: $\underline{10.17675 / 2305-6894-2021-10-2-10}$ 


\section{Introduction}

Corrosion inhibitors have been used to protect mild steel surface and other alloys from corrosion. Corrosion inhibitors were considered to be an excellent technique to protect alloys from corrosive solutions [1]. Corrosion inhibitors are natural or synthesized compounds added at certain concentrations to acid or alkaline environments in order to reduce metals corrosion [2]. Many corrosion inhibitors are organic molecules [3]; it is mainly supposed that corrosion inhibition is accomplished by adsorption of molecules on the surface of the metal [4]. The structure of the inhibitor molecule is an important indicator in knowing the inhibitive performance [5]. It has been reported that efficient corrosion inhibitors should have $\mathrm{O}, \mathrm{N}, \mathrm{S}$, and/or $\mathrm{P}$ atoms in their structure. These atoms have unpaired electrons that aid in the corrosion protection of alloys. Similarly, it has been reported that compounds with a $\pi$-conjugate association system have the potential to inhibit corrosion. Corrosion is worth researching in light of the fact that corrosion problems represent a large portion of the expenditures in industries each year around the world [6]. This is an issue for nearly every manufacture that deals with metal structures extending from transport, petrol to construction industries [7]. Corrosion urges the automatic closure of plants and causes horrific accidents which ultimately lead to damage environment and human injuries [8]. It has been explained that $25 \%$ of deaths in the petrol manufactures are generally due to corrosion. Over the decades, efficient methods to reduce and/or impede the corrosion have been published by a considerable number of researchers. In particular, this includes cathodic/anodic protection, application of coatings, and corrosion inhibitors [9]. Most methods of preventing corrosion have their own drawbacks; but among these techniques, it has been shown that the use of corrosion inhibitors is the most efficient technique [10] to protect metals in corrosive environments [11]. This indicates that the utilization of corrosion inhibitors to reduce/impede the corrosion rate is clearly exceptionally important in pipeline manufactures. To expand the scope of novel efficient inhibitors [12-18], the objective of this investigation was to synthesize, characterize and study the inhibitive effects of a new Schiff base, namely, 5-((4-fluorobenzylidene)amino)1,3,4-thiadiazole-2-thiol (FATT), for mild steel in a hydrochloric acid environment using the gravimetric technique. The corrosion inhibition performance of FATT molecules in $1.0 \mathrm{M}$ hydrochloric acid and the relationship with some quantum chemical factors such as HOMO, LUMO, $\Delta E, \mu, \chi, \eta, \sigma$ and $\Delta N$ have been investigated. The methodological inhibition efficiency and DFT calculations ought to correlate in order to confirm the accuracy of the suggest theoretical method for assessment of the inhibitive effects of the new inhibitor. Figure 1 demonstrates the structure of the new corrosion inhibitor.

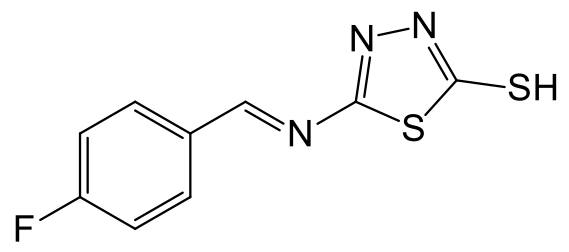

Figure 1. Molecular structure of the investigated inhibitor. 


\section{Experimental}

\subsection{Chemistry}

All chemicals were purchase from Sigma-Aldrich Malaysia and were used as received without further purification. Proton nuclear magnetic resonance $\left({ }^{1} \mathrm{H}\right.$ NMR) and Carbon-13 nuclear magnetic resonance $\left({ }^{13} \mathrm{C} \mathrm{NMR}\right)$ spectra were recorded at room temperature on a $600 \mathrm{MHz}$ spectrometer (Bruker, Billerica, MA, USA), in DMSO-d $\mathrm{d}_{6}$, using TMS as the internal standard. IR spectra were recorded on a Nicolet 6700 FT-IR spectrophotometer (Thermo Nicolet Corp., Madison, WI, USA), in the $4000-400 \mathrm{~cm}^{-1}$ region using $\mathrm{KBr}$ pellets. The chemical elemental analysis for the determination of $\mathrm{C}, \mathrm{H}$ and $\mathrm{N}$ was performed with an Elementar Vario El III Carlo Erba 1108 elemental analyzer (Carlo Erba Reagenti SpA, Rodano, Italy).

\subsection{Synthesis of 5-((4-fluorobenzylidene)amino)-1,3,4-thiadiazole-2-thiol (FATT) as a} corrosion inhibitor

A mixture of equimolar amounts 4-fluorobenzaldehyde and 5-amino-1,3,4-thiadiazole-2thiol in ethanol was refluxed for $6 \mathrm{~h}$ and cooled. The resulting precipitate was filtered off. The precipitate was washed with ether and recrystallized from ethanol. M.P. $233.5^{\circ} \mathrm{C}$. Fourier transform infrared (FT-IR) spectrum: $3022.7(\mathrm{C}-\mathrm{H}$ aromatic), $1611.4(\mathrm{C}=\mathrm{N})$, $1598.6(\mathrm{C}=\mathrm{C}) .{ }^{1} \mathrm{H}$ NMR: $\delta_{\mathrm{H}} 12.95(\mathrm{~s}, 1 \mathrm{H}, \mathrm{S}-\mathrm{H}), 8.77(\mathrm{~s}, 1 \mathrm{H}, \mathrm{CH}=\mathrm{N}), 7.44(\mathrm{dd}, 2 \mathrm{H}$, $\mathrm{H}$ aromatic) and $7.86\left(\mathrm{dd}, 2 \mathrm{H}, \mathrm{H}\right.$ aromatic). ${ }^{13} \mathrm{C} \mathrm{NMR}: \delta_{\mathrm{C}} 166.8(\mathrm{~S}-\mathrm{C}=\mathrm{N}), 164.3(\mathrm{C}-\mathrm{F})$, $162.9(\mathrm{~N}-\mathrm{C}=\mathrm{N}), 157.8(\mathrm{CH}=\mathrm{N}), 133.4(\mathrm{C}=\mathrm{C}$ aromatic $), 130.0(\mathrm{C}=\mathrm{C}$ aromatic $), 115.9$ $\left(\mathrm{C}=\mathrm{C}\right.$ aromatic). Anal. Calcd for: $\mathrm{C}_{9} \mathrm{H}_{6} \mathrm{FN}_{3} \mathrm{~S}_{2}, \mathrm{C}, 45.17 ; \mathrm{H}, 2.53 ; \mathrm{N}, 17.56 \%$. Found: $\mathrm{C}$, $45.37 ; \mathrm{H}, 2.44 ; \mathrm{N}, 16.81$.

\subsection{Materials}

Mild steel coupons (Metal Samples Company) with the chemical composition: iron, 99.21; carbon, 0.210; silicon, 0.380; phosphorous, 0.090; S, 0.05; manganese, 0.050; and aluminum, 0.010 , were used. The coupons utilized for gravimetric techniques were cut as $2.0 \times 1.0 \times 0.05 \mathrm{~cm}^{3}$ pieces. The corrosive solution of $1 \mathrm{M}$ hydrochloric acid was prepared by dilution of $37 \% \mathrm{HCl}$ with distilled water.

\subsection{Weight loss technique}

Weight loss experiments were performed by immersing the specimens of mild steel into the corrosive environment in the absence and presence of various concentrations of FATT as the corrosion inhibitor. This study was performed at inhibitor concentrations of $0,0.1,0.2,0.3$, 0.4 , and $0.5 \mathrm{mM}$; at temperatures of $303,313,323$ and $333 \mathrm{~K}$; and with immersion times of 1, 5, 10 and $24 \mathrm{~h}$. Before all measurements, the mild steel coupons were mechanically polished with various emery papers, rinsed with distilled water, degreased with acetone, dried at room temperature, and weighed by following the method mentioned in reference [19] in compliance with the standard procedure of ASTM, G1-03. The corrosion rate $\left(C_{R}\right)$ in 
$\mathrm{mg} \mathrm{cm}^{-2} \mathrm{~h}^{-1}$, inhibition efficiency (IE\%) and surface coverage $(\theta)$ were determined according to Equations (1)-(3).

$$
\begin{gathered}
C_{R}=\frac{W_{0}-W_{\mathrm{i}}}{S_{\text {area of coupon }} \times t_{\text {immersion }}} \\
I E \%=\frac{W_{0}-W_{\mathrm{i}}}{W_{0}} \times 100 \\
\theta=\frac{W_{0}-W_{\mathrm{i}}}{W_{0}}
\end{gathered}
$$

Where $W_{0}$ and $W_{\mathrm{i}}$ are the values of weight loss without and with FATT, respectively. Equation 3 is only valid for inhibitors with blocking action.

\subsection{Theoretical calculations}

Density function theory calculations were performed using DFT/B3LYP implemented with the 6-31G basis set. DFT has been constantly adopted to calculate the details of the molecular structure and distributions of the electrons and utilized quite often to analyze the inhibitive performance and interactions of the inhibitor molecules with metals due to its accuracy and lack of time requirements from the mathematical point of view [20, 21]. The ionization potential $(I)$ and electron affinity $(A)$ can be calculated according to the Koopman theory. This theory confirms the relationship between the HOMO and LUMO energies, the potential $I$ and $A$, respectively. Although there is no formal evidence for this theory within DFT, its validity is mostly reasonable. The ionization potential and electron affinity are linked in turn to HOMO and LUMO by Equations 4 and 5:

$$
\begin{aligned}
& I=-E_{\text {HOMO }} \\
& A=-E_{\text {LUMO }}
\end{aligned}
$$

The $\chi$ and $\eta$ values of the FATT molecule are shown in Equations 6 and 7.

$$
\begin{gathered}
\chi=\frac{I+A}{2} \\
\eta=\frac{I-A}{2}
\end{gathered}
$$

The $\sigma$ can be calculated according to Equation 8:

$$
\sigma=\frac{1}{\eta}
$$

The $\Delta N$ from the FATT molecule to the iron atom was calculated according to Equation 9,

$$
\Delta N=\frac{\chi_{\mathrm{Fe}}-\chi_{\mathrm{inh}}}{2\left(\eta_{\mathrm{Fe}}+\eta_{\mathrm{inh}}\right)}
$$

where, $\chi_{\mathrm{Fe}}=7 \mathrm{eV}$ and $\eta_{\mathrm{Fe}}=0$, under the assumption that $I=A$ for a metal [22]. 


\section{Results and Discussion}

\subsection{Chemistry}

The reaction sequence for the synthesis of FATT starting from thiosemicarbazide is outlined in Figure 2.
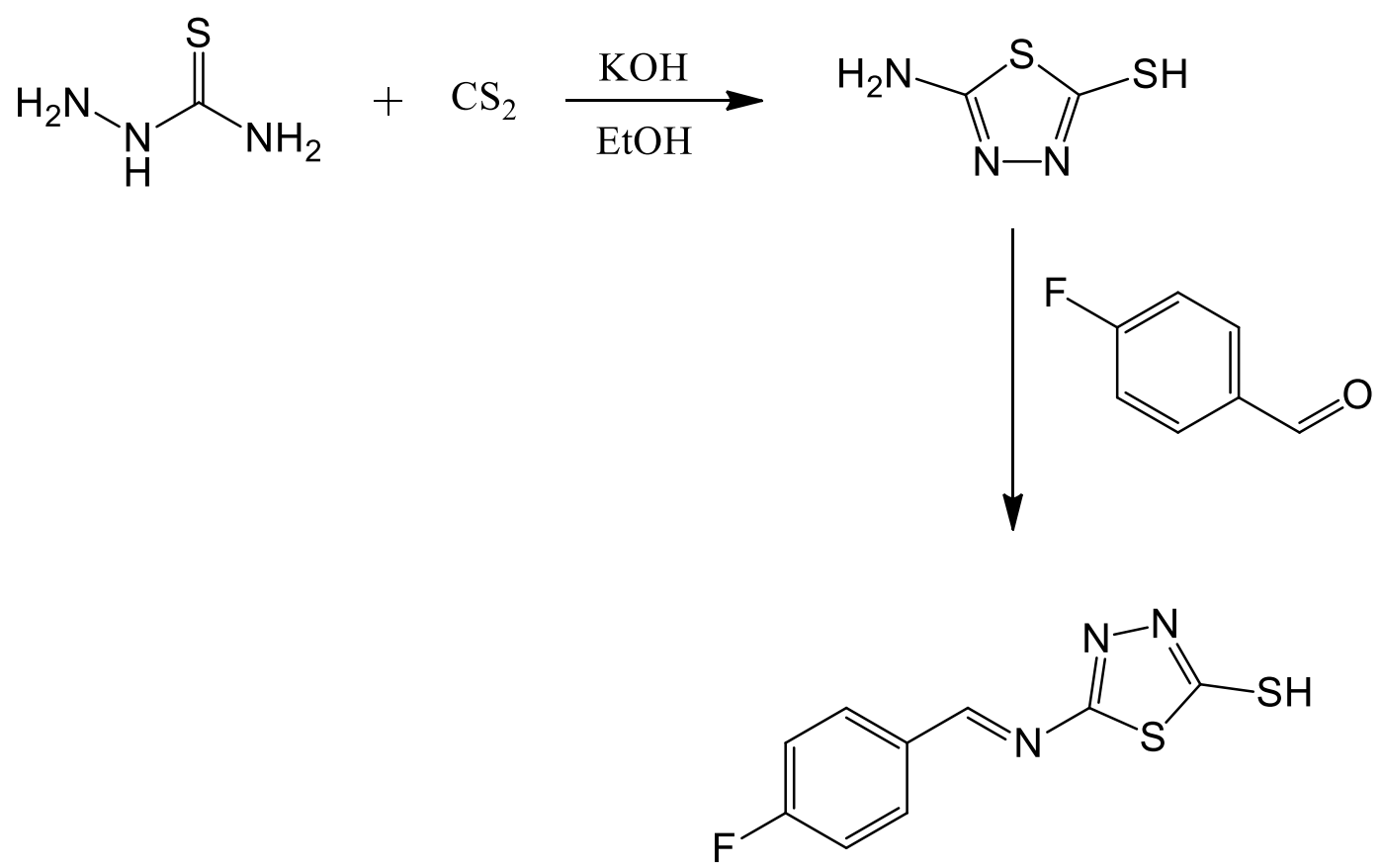

Figure 2. Reaction sequence for the synthesis of FATT.

The reaction of thiosemicarbazide with carbon disulfide in the presence of alcoholic potassium hydroxide yielded 5-amino-1,3,4-thiadiazole-2-thiol as a precursor for the synthesis of FATT. The new corrosion inhibitor 5-((4-fluorobenzylidene)amino)-1,3,4thiadiazole-2-thiol (FATT) was synthesized by the reaction of 5-amino-1,3,4-thiadiazole-2thiol with 4-fluorobenzaldehyde. The FT-IR spectrum of FATT showed an absorption band at $3022.71 \mathrm{~cm}^{-1}\left(\mathrm{C}-\mathrm{H}\right.$, aromatic stretching) and $1611.4 \mathrm{~cm}^{-1}$ for $\mathrm{C}=\mathrm{N}$. The ${ }^{1} \mathrm{H} \mathrm{NMR}$ spectrum exhibited a singlet at $\delta 12.95 \mathrm{ppm}$ due to the $\mathrm{SH}$ proton. A singlet at $\delta 8.77 \mathrm{ppm}$ is due to the $\mathrm{CH}$ proton of $\mathrm{CH}=\mathrm{N}$ - and a multiplet is due to the aromatic protons at $\delta 7.44(\mathrm{dd}$, $2 \mathrm{H}, \mathrm{H}$ aromatic) and $7.86(\mathrm{dd}, 2 \mathrm{H}, \mathrm{H}$ aromatic).

\subsection{Weight loss measurements}

The effect of FAAT at concentrations of $0,0.1,0.2,0.3,0.4$, and $0.5 \mathrm{mM}$ on mild steel corrosion in the corrosive environment was studied by the weight loss technique at temperatures of $303,313,323$ and $333 \mathrm{~K}$, for immersion times of 1, 5, 10 and $24 \mathrm{~h}$. The corrosion rates (CR) and inhibition efficiencies (IE\%) as corrosion parameters at various concentrations of FATT are demonstrated in Figures 3 and 4. 


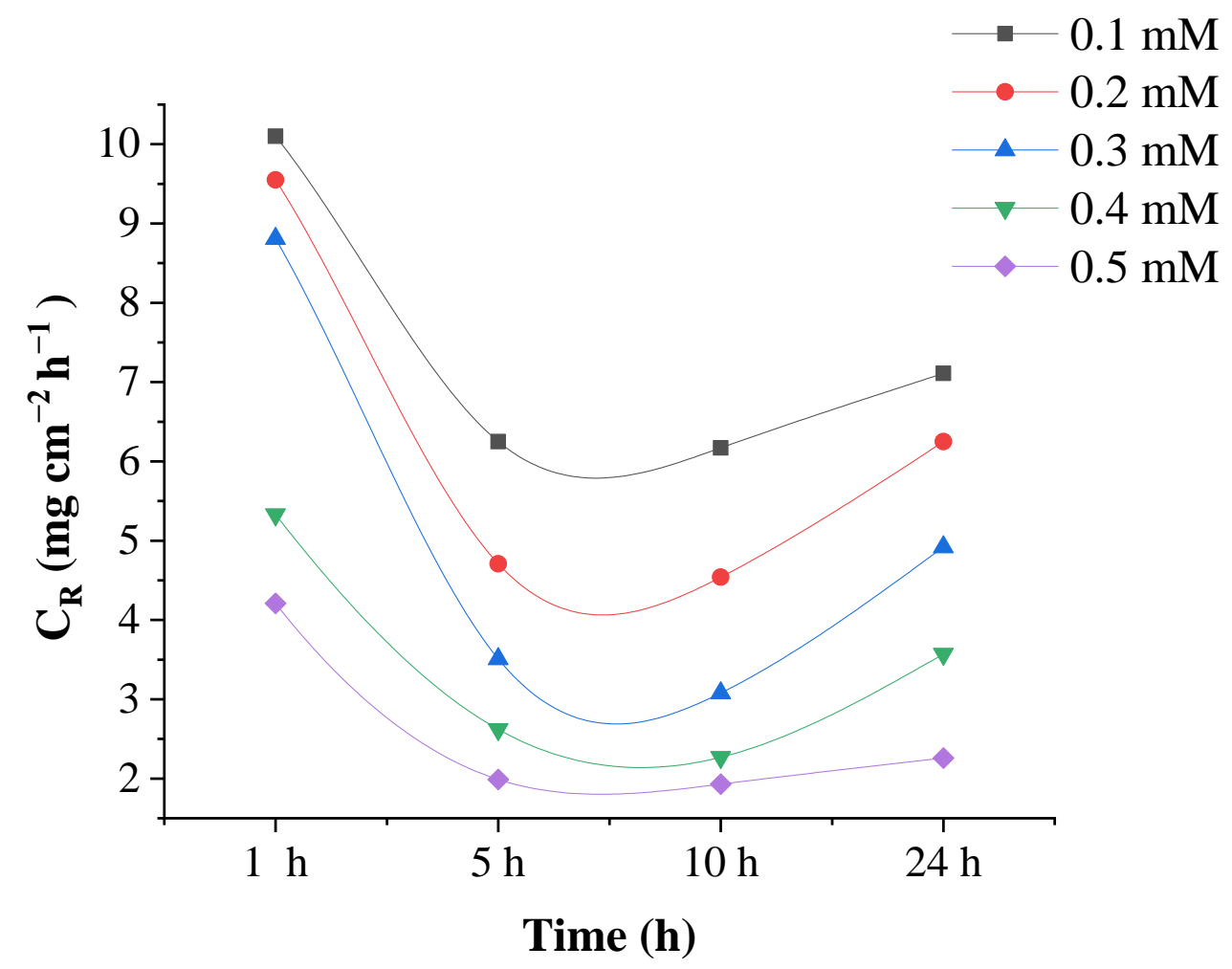

Figure 3. Corrosion rates of mild steel in $1.0 \mathrm{M} \mathrm{HCl}$ in the presence of various concentrations of FATT measured by the weight loss technique at $303 \mathrm{~K}$ for $1-24 \mathrm{~h}$ time periods.

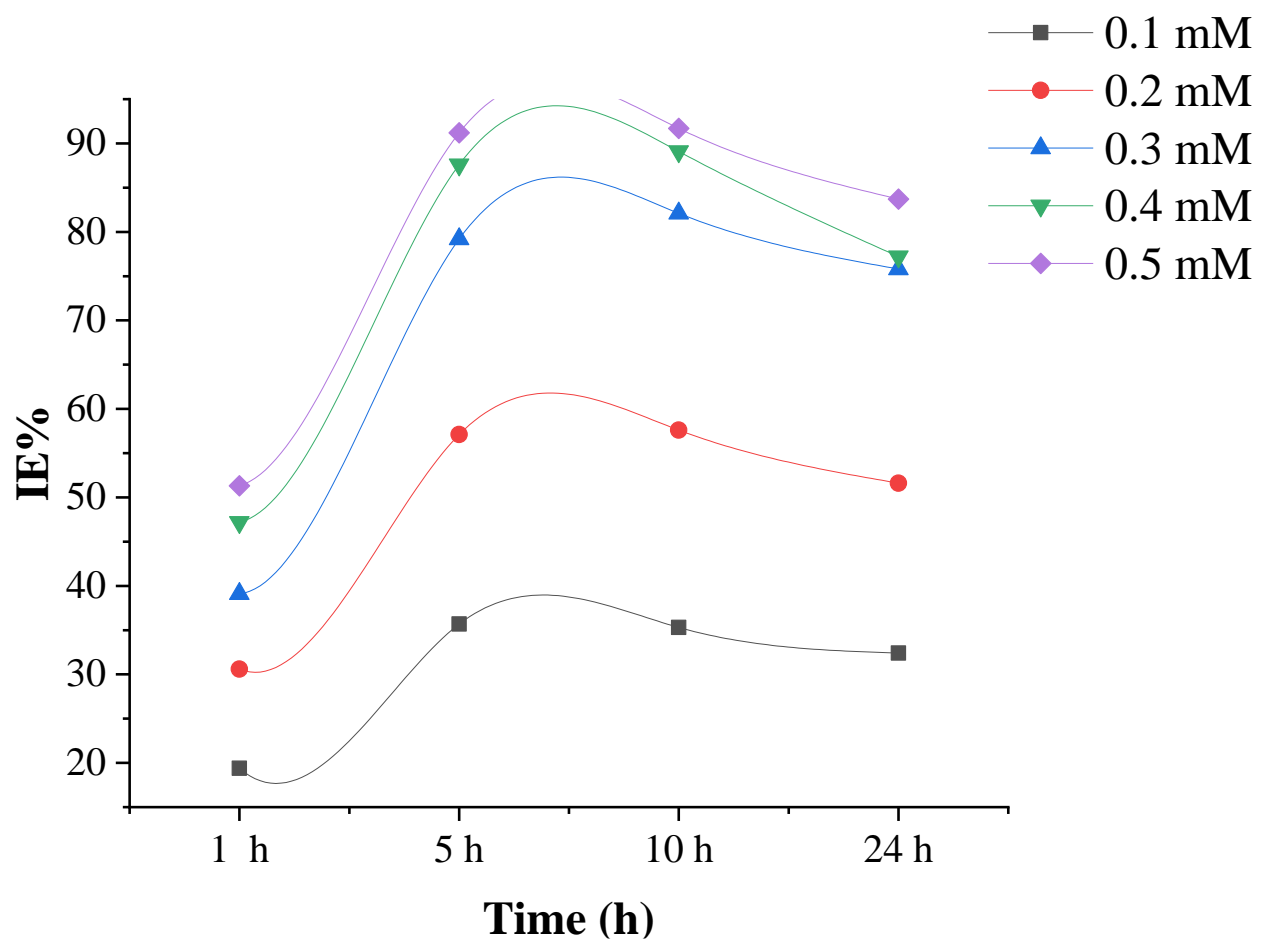

Figure 4. Inhibition efficiencies of mild steel in $1.0 \mathrm{M} \mathrm{HCl}$ in the presence of various concentrations of FATT measured by the weight loss technique at $303 \mathrm{~K}$ for $1-24 \mathrm{~h}$ time periods. 
As demonstrated in Figures 3 and 4, the tested inhibitor has the ability to inhibit the corrosion of mild steel at all studied concentrations in 1.0 M hydrochloric acid environment and the inhibitive performance is obvious at higher concentration. An examination of this data shows an increase in inhibition performance and a decrease in corrosion rate with an increase in the concentration of the tested inhibitor. This action can be imputed to an increase in the adsorption of FATT molecules on mild steel surface with an increase in the inhibitor concentration. It is also clear that FATT is an excellent corrosion inhibitor at higher concentrations studied. The inhibition efficiency can be explained by the functional group substituents on the benzene ring of the FATT molecule and 1,3,4-thiadiazole ring in addition to the presence of heteroatoms and a $\mathrm{C}=\mathrm{N}$ group.

\subsection{Effect of Temperature}

To investigate the effect of temperature on the corrosion of mild steel surface in the corrosive solution, gravimetric measurements were performed at 303.0, 313.0, 323.0 and $333.0 \mathrm{~K}$ with addition of various concentrations of FATT as a corrosion inhibitor and with an immersion time of 5.0 hours. The effect of temperature on the inhibitive performance is demonstrated in Figure 5. The inhibitive performance decreases with increasing temperature imputed to the absorption of FATT molecules on the investigated coupon surface with an increase in temperature from $303.0 \mathrm{~K}$ to $333.0 \mathrm{~K}$.

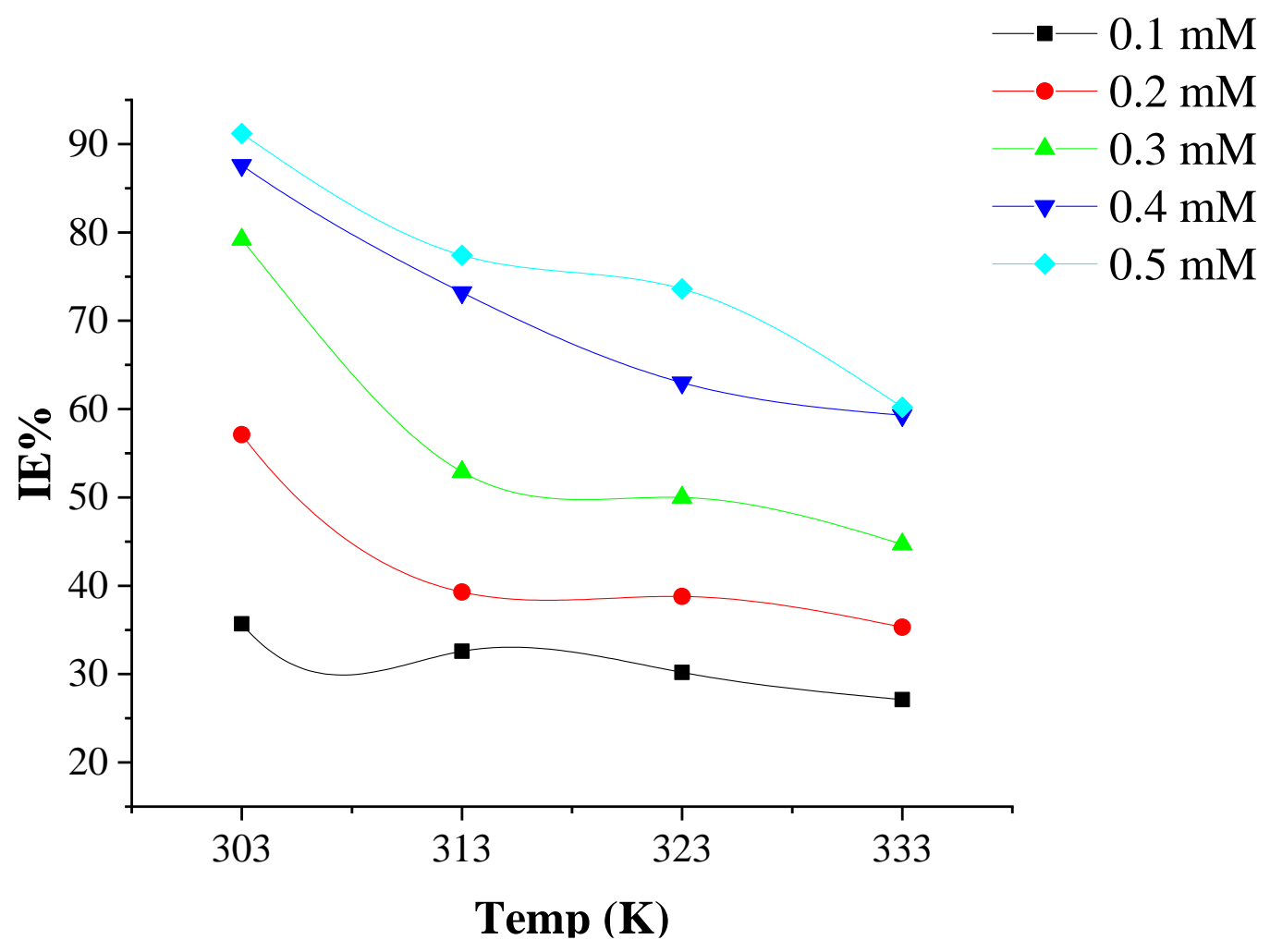

Figure 5. Inhibition efficiency for mild steel in $1.0 \mathrm{M} \mathrm{HCl}$ in the presence of various concentrations of inhibitor (FATT) from gravimetric measurements at various temperatures. 


\subsection{Adsorption Isotherms}

The values of surface coverage $(\theta)$ for FATT were employed to determine the adsorption isotherms and to understand the interactions of the FATT molecules with mild steel surface. FATT molecules are adsorbed on mild steel surface either due to a chemical reaction or by physical interaction. To understand this phenomenon, adsorption isotherms such as Langmuir isotherm, Temkin isotherm and Freundlich isotherm were studied. The Langmuir isotherm is the best one. The intercept value for Langmuir adsorption is $-0.0737 \pm 0.01737$ and the slope value is $1.0927 \pm 0.04753$. The $C / \theta$ and $C$ plot is demonstrated in 6 and calculated as follows in equations 10 and 11:

$$
\frac{C}{\theta}=\frac{1}{K_{\text {ads }}}+C
$$

where $C$ is the concentration FATT, and $\theta$ is the surface coverage, and $K_{\text {ads }}$ is the adsorption constant;

$$
\Delta G_{\text {ads }}=-R T \ln \left(55.5 K_{\text {ads }}\right)
$$

whereas $\Delta G_{\text {ads }}$ is the free energy of adsorption, and the value of 55.5 is the molar water concentration, $R$ is the gas constant, and $T$ is the absolute temperature.

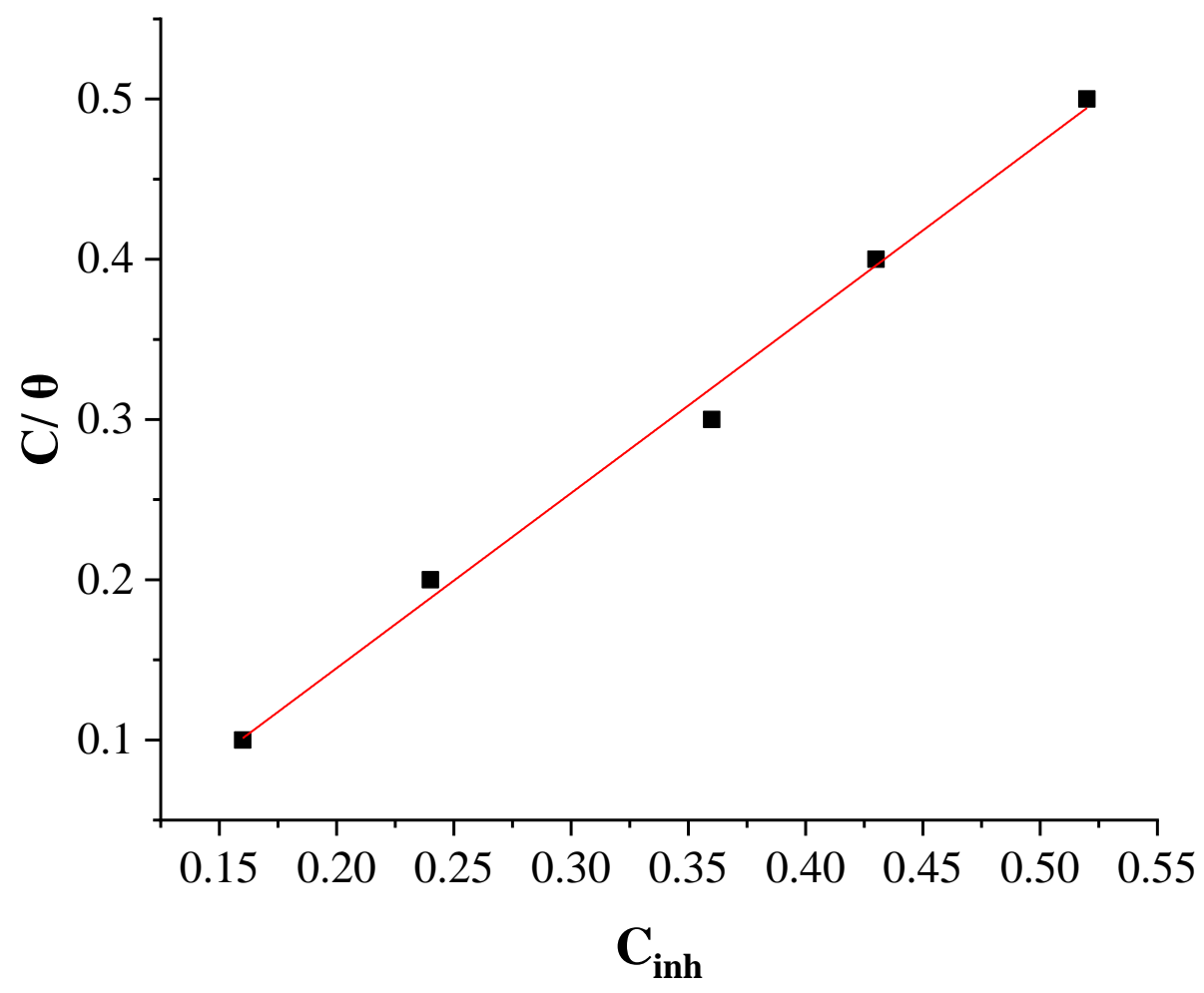

Figure 6. Langmuir adsorption isotherm of mild steel in the presence of different concentrations of FATT. 
Generally, the values of $\Delta G_{\text {ads }}$ around $-40 \mathrm{~kJ} / \mathrm{mol}$ indicate adsorption of the type chemisorption, but if the value of $\Delta G_{\text {ads }}$ is around $-20 \mathrm{~kJ} / \mathrm{mol}$ or less negative, adsorption of the type physisorption is suggested [21-23]. The $\Delta G_{\text {ads }}$ value for FATT, which is $35.25 \mathrm{~kJ} / \mathrm{mol}$, suggests that adsorption of FATT molecules on mild steel surface follows Langmuir adsorption isotherm. The adsorption of FATT molecules on mild steel surface is mixed adsorption involving both physisorption and chemisorption.

\subsection{Scanning electron microscopy}

The morphology of coupon surface was determined from SEM images. In the absence of the inhibitor, it was corroded by the corrosive solution as demonstrated in Figure 7a. It was found that the corroded coupon surface had a number of pits. The SEM image of the coupon surface in the presence of FATT molecules was observed to be smooth as shown in Figure 7b. This observation implies that the mild steel surface is not damaged, indicating that the inhibitor (FATT) inhibits corrosion, i.e., controls or retards the attack of the corrosive solution and dissolution of the tested coupon surface and thus reduces the corrosion rate.
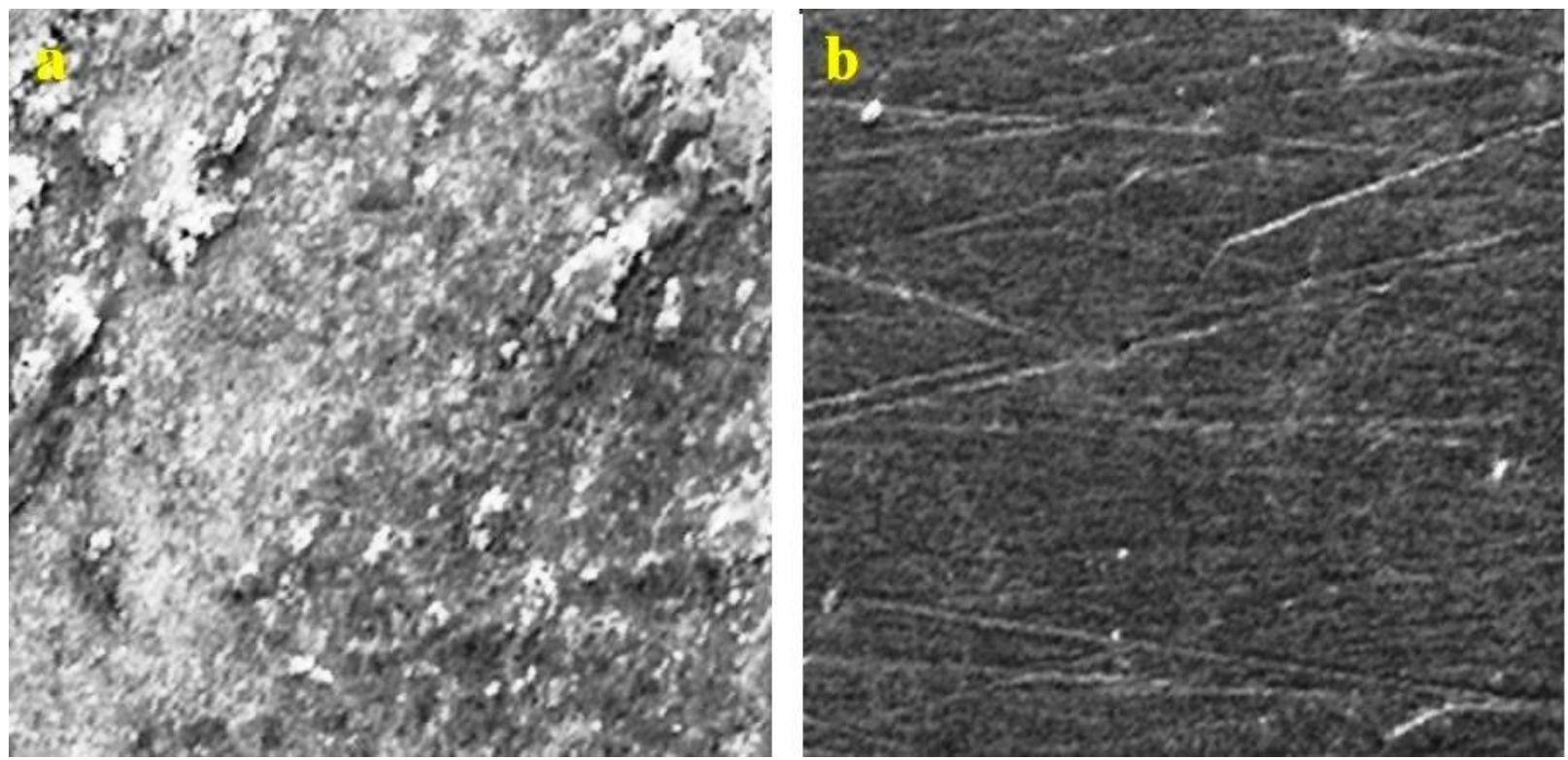

Figure 7. SEM images of the samples after exposure to $1 \mathrm{M} \mathrm{HCl}$ solution (a) without an inhibitor and (b) in the presence of $0.5 \mathrm{mM}$ FATT.

\subsection{Quantum-chemical parameters}

The optimized structure, highest occupied molecular orbital (HOMO) and lowest unoccupied molecular orbital (LUMO) of the FATT molecule in aqueous phase are demonstrated in Figure 8. HOMO provides data about the molecule sites which donate electrons to the unoccupied d-orbital of the surface atoms of mild steel (iron atoms). LUMO electron density demonstrates the sites accepting electrons from donor molecules which have unpaired electrons or $\pi$-bonds. $E_{\mathrm{HOMO}}$ is related with the susceptibility of donating electrons, 
while $E_{\mathrm{LUMO}}$ is related with the accepting electrons. Thus, $E_{\text {Hомо }}$ with high value signifies a high tendency to donate electrons from the inhibitor molecules to the empty metal d-orbital. Furthermore, $E_{\text {LUMo }}$ with low values indicates an easier inhibitor tendency to accept electrons from unpaired electrons of the metal surface. DFT parameters of the FATT molecules are listed in Table 2 along with inhibition efficiency obtained experimentally.

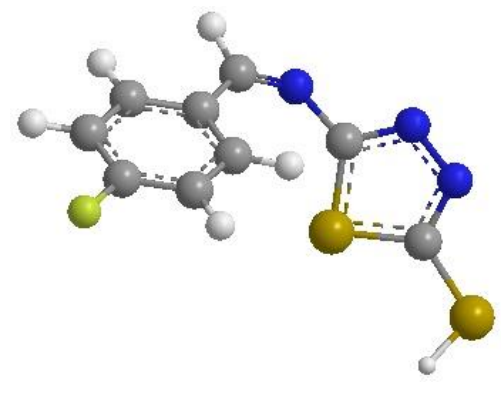

Optimized structure

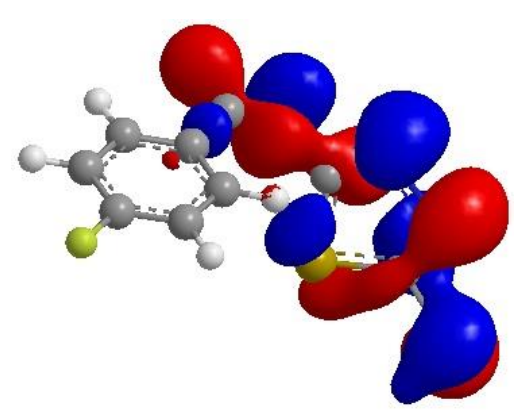

HOMO

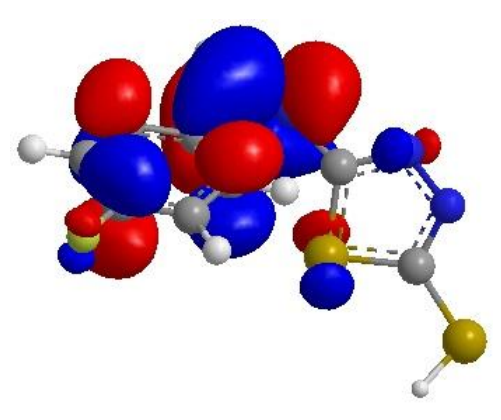

LUMO

Figure 8. HOMO, LUMO, and optimized molecular structure for the tested corrosion inhibitor.

Table 1. Quantum Chemical Parameters for the tested corrosion inhibitor.

\begin{tabular}{cc}
\hline Quantum Chemical Parameters & Values \\
\hline HOMO $(\mathrm{eV})$ & -8.03 \\
LUMO $(\mathrm{eV})$ & $-2.38 \mathrm{eV}$ \\
$\Delta E(\mathrm{eV})$ & $-5.65 \mathrm{eV}$ \\
$\eta(\mathrm{eV})$ & 2.82 \\
$\sigma(\mathrm{eV})$ & 0.35 \\
$\chi(\mathrm{eV})$ & -5.34 \\
$I$ & 8.03 \\
$A$ & 2.38 \\
$\Delta N$ & 4.15 \\
$I E(\%)$ & 91 \\
\hline
\end{tabular}

FATT molecules have a high HOMO value imputed to the substituents on the benzene ring and the presence of 1,3,4-thiadiazole. The $\mathrm{C}=\mathrm{N}$ and $\mathrm{SH}$ groups contribute towards a higher HOMO value, which suggests that FATT molecules show a significant susceptibility to donate electrons. The LUMO of FATT molecules shows a significant tendency to accept electrons. $\Delta E$ with a moderate value confirms the high susceptibility to adsorb FATT molecules on the surface of mild steel coupon. The absolute electronegativity $(\chi)$ defines the 
electron attraction ability of a group of atoms toward itself. The higher value of $\eta$ indicates the low susceptibility of electron transfer. The $\sigma$ presents the significant susceptibility of inhibitor molecules to undergo reactions. A hard molecule has a large $\Delta E$ and a soft molecule has a small $\Delta E[21,24]$. Therefore, $\Delta E$ is associated with the global hardness. This association depends on the standard of significant hardness, that is "the molecule organizes itself to be hard". From Table 1, it is demonstrated that the FATT molecules have the lowest value of $\chi$, which suggests a significant tendency to donate electrons. The low value of $\eta$ and the significant value of $\sigma$ suggest the excellent reactivity of FATT molecules. These results are in good agreement with the $\Delta E$ value demonstrated above. The fraction of electrons transferred does not provide an accurate number of electrons transferred from the inhibitor molecules and the metal surface, but suggested that the transfer of electrons occurs from the inhibitor on to the metal surface. The theoretical values of $\chi_{\mathrm{Fe}}=7.0 \mathrm{eV}$ and $\eta_{\mathrm{Fe}}=0$ are used to calculate the number of electrons transferred [19-22]. The theoretical calculated value of $\Delta N$ is $4.51 \mathrm{eV}$ as shown in Table 1. It is reported that if $\Delta N>0$, then the electrons will be transferred from the inhibitor to the metal surface. Thus, it is obvious that the trend of calculated quantum chemical parameters matches the tendency of inhibition performance determined experimentally.

\subsubsection{Mulliken charge}

Analyses of Mulliken charge have been done in order to determine the site existing in the FATT molecules which are the most possible donor-acceptor sites for interactions with the metal surface. The charge of an atom is determined by the type of molecule in that it donates and accepts electrons by nucleophilic (negative charge) and/or electrophilic (positive charge) sites, respectively. A higher nucleophilic or electrophilic value represents a significant ability to donate or accept electrons, respectively. From Figure 9 and Table 2 it can be shown that the most susceptible sites for electron donation in FATT molecules are $C(2), N(4)$ and $N(7)$, whereas the sites most susceptible for electron acceptance are $S(1), S(6)$ and $N(3)$.

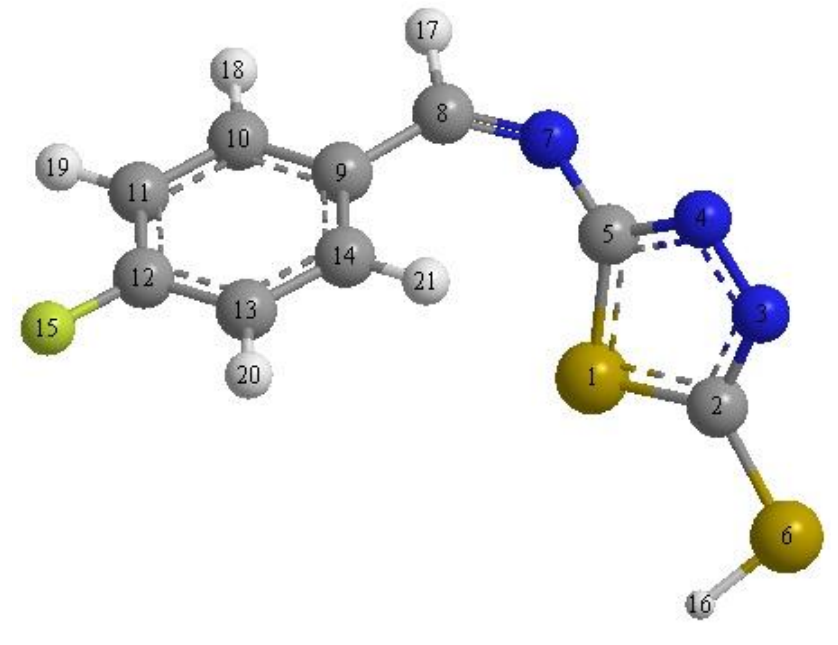

Figure 9. The atomic numbers of FATT molecule. 
Table 2. Mulliken charge of FATT Molecules

\begin{tabular}{cccccccc}
\hline Atom & Charge & Atom & Charge & Atom & Charge & Atom & Charge \\
\hline $\mathrm{S}(1)$ & 0.2637 & $\mathrm{~S}(6)$ & 0.2038 & $\mathrm{C}(11)$ & -0.1403 & $\mathrm{H}(16)$ & 0.0052 \\
$\mathrm{C}(2)$ & -0.3874 & $\mathrm{~N}(7)$ & -0.0059 & $\mathrm{C}(12)$ & 0.093 & $\mathrm{H}(17)$ & 0.1121 \\
$\mathrm{~N}(3)$ & 0.0323 & $\mathrm{C}(8)$ & 0.0369 & $\mathrm{C}(13)$ & -0.1367 & $\mathrm{H}(18)$ & 0.1111 \\
$\mathrm{~N}(4)$ & -0.0829 & $\mathrm{C}(9)$ & -0.1356 & $\mathrm{C}(14)$ & -0.0436 & $\mathrm{H}(19)$ & 0.128 \\
$\mathrm{C}(5)$ & -0.1831 & $\mathrm{C}(10)$ & -0.0388 & $\mathrm{~F}(15)$ & -0.0856 & $\mathrm{H}(20)$ & 0.1302 \\
\hline
\end{tabular}

\section{Conclusions}

- The synthesized novel Schiff base, namely 5-((4-fluorobenzylidene)amino)-1,3,4thiadiazole-2-thiol (FATT), demonstrated an excellent inhibition performance for the corrosion of mild steel in $0.1 \mathrm{M}$ hydrochloric acid solution. The $I E$ increased with increasing FATT concentration, and the maximum inhibition efficiency of $91 \%$ was obtained at $0.5 \mathrm{mM}$ using the weight loss technique.

- The adsorption of FATT molecules on the tested coupon surface obeys the Langmuir adsorption isotherm.

- A film was formed on the surface of tested coupons as illustrated by SEM.

- Quantum chemical calculations using density functional theory (DFT) were employed to identify the relationship between the electronic structure parameters of the FATT molecule and inhibition efficiency. It was found that the theoretical calculations are in good agreement with experimental work.

\section{Conflict of Interests}

The authors declare there is no conflict of interests.

\section{Acknowledgments}

The authors are grateful to University of Technology/Baghdad-Iraq and Universiti Kebangsaan Malaysia (GUP-2019-045) for support.

\section{References}

1. S.S. Al-Taweel, K.W.S. Al-Janabi, H.M. Luaibi, A.A. Al-Amiery and T.S. Gaaz, Evaluation and characterization of the symbiotic effect of benzylidene derivative with titanium dioxide nanoparticles on the inhibition of the chemical corrosion of mild steel, Int. J. Corros. Scale Inhib., 2019, 8, no. 4, 1149-1169. doi: 10.17675/2305-6894-2019$\underline{8-4-21}$

2. A. Mohamad, A. Kadhum, A. Al-Amiery, L. Ying and A. Musa, Synergistic of a coumarin derivative with potassium iodide on the corrosion inhibition of aluminum alloy in 1.0 $\mathrm{M} \mathrm{H}_{2} \mathrm{SO}_{4}$, Met. Mater. Int., 2014, 20, 459-467. doi: 10.1007/s12540-014-3008-3 
3. T.A. Salman, D.S. Zinad, S.H. Jaber, M. Al-Ghezi, A. Mahal, M.S. Takriff and A.A. AlAmiery, Effect of 1,3,4 Thiadiazole Scafold on the Corrosion Inhibition of Mild Steel in Acidic Medium: An Experimental and Computational Study, J. Bio Tribo Corrosion, 2019, 5, 1-11. doi: 10.1007/s40735-019-0243-7

4. A. Kadhim, A. Al-Okbi, D.M. Jamil, A. Qussay, A.A. Al-Amiery, T.S. Gaaz, A.A.H. Kadhum, A.B. Mohamad and M.H. Nassir, Experimental and theoretical studies of benzoxazines corrosion inhibitors, Results Phys., 2017, 7, 4013-4019. doi: 10.1016/j.rinp.2017.10.027

5. K.F. Al-Azawi, I.M. Mohammed, S.B. Al-Baghdadi, T.A. Salman, H.A. Issa, A.A. AlAmiery, T.S. Gaaz and A.A.H. Kadhum, Experimental and quantum chemical simulations on the corrosion inhibition of mild steel by 3-((5-(3,5-dinitrophenyl)-1,3,4thiadiazol-2-yl)imino)indolin-2-one, Results Phys., 2018, 9, 278-283. doi: 10.1016/j.rinp.2018.02.055

6. S.B. Al-Baghdadi, F.G. Hashim, A.Q. Salam, T.K. Abed, T.S. Gaaz, A.A. Al-Amiery, A.A.H. Kadhum, K.S. Reda and W.K. Ahmed, Synthesis and corrosion inhibition application of NATN on mild steel surface in acidic media complemented with DFT studies, Results Phys., 2018, 8, 1178-1184. doi: 10.1016/j.rinp.2018.02.007

7. D.S. Zinad, Q.A. Jawad, M.A.M. Hussain, A. Mahal, L. Mohamed and A.A. AlAmiery. Adsorption, temperature and corrosion inhibition studies of a coumarin derivatives corrosion inhibitor for mild steel in acidic medium: gravimetric and theoretical investigations, Int. J. Corros. Scale Inhib., 2020, 9, no. 1, 134-151. doi: 10.17675/2305-6894-2020-9-1-8

8. D.S. Zinad, M. Hanoon, R.D. Salim, S.I. Ibrahim, A.A. Al-Amiery, M.S. Takriff and A.A.H. Kadhum. A new synthesized coumarin-derived Schiff base as a corrosion inhibitor of mild steel surface in $\mathrm{HCl}$ medium: gravimetric and DFT studies, Int. J. Corros. Scale Inhib., 2020, 9, no. 1, 228-243. doi: 10.17675/2305-6894-2020-9-1-14

9. D.M. Jamil, A.K. Al-Okbi， S.B. Al-Baghdadi, A.A. Al-Amiery, A. Kadhim and T.S. Gaaz, Experimental and theoretical studies of Schiff bases as corrosion inhibitors, Chem. Cent. J., 2018, 12, 1-7. doi: 10.1186/s13065-018-0376-7

10. J.A.A. Yamin, E. Ali Eh Sheet and A. Al-Amiery, Statistical analysis and optimization of the corrosion inhibition efficiency of a locally made corrosion inhibitor under different operating variables using RSM, Int. J. Corros. Scale Inhib., 2020, 9, no. 2, 502-518. doi: $10.17675 / 2305-6894-2020-9-2-6$

11. A.A. Al-Amiery, L.M. Shaker, A.A.H. Kadhum, M.S. Takriff. Corrosion Inhibition of Mild Steel in Strong Acid Environment by 4-((5,5-dimethyl-3-oxocyclohex-1-en-1yl)amino)benzenesulfonamide, Tribology in Industry, 2020, 42, no. 1, 89-101. doi: $\underline{10.24874 / \text { ti.2020.42.01.09 }}$

12. A. Al-Amiery, T.A. Salman, K.F. Alazawi, L.M. Shaker, A.H. Kadhum and M.S. Takrif. Quantum chemical elucidation on corrosion inhibition efficiency of Schiff base: DFT investigations supported by weight loss and SEM techniques, Int. J. Low-Carbon Technol., 2020, 15, 202-209. 
13. H.J. Habeeb, H.M. Luaibi, R.M. Dakhil, A.A.H. Kadhum, A.A. Al-Amiery and T.S. Gaaz, Development of new corrosion inhibitor tested on mild steel supported by electrochemical study, Results Phys., 2018, 8, 1260-1267. doi: 10.1016/j.rinp.2018.02.015

14. A. Al-Amiery, A. Kadhum, A. Mohamad, A. Musa and C. Li, Electrochemical study on newly synthesized chlorocurcumin as an inhibitor for mild steel corrosion in hydrochloric acid, Materials, 2013, 6, 5466-5477. doi: 10.3390/ma6125466

15. S. Junaedi, A. Kadhum, A. Al-Amiery, A. Mohamad and M. Takriff, Synthesis and characterization of novel corrosion inhibitor derived from oleic acid: 2-Amino5Oleyl1,3,4-Thiadiazol (AOT), Int. J. Electrochem. Sci., 2012, 7, 3543-3554.

16. T.A. Salman, Q.A. Jawad, M.A. Hussain, A. Al-Amiery, L. Shaker, A. Kadhum, M. and Takriff, M.S., Novel ecofriendly corrosion inhibition of mild steel in strong acid environment: Adsorption studies and thermal effects, Int. J. Corros. Scale Inhib., 2019, 8, no. 4, 1123-1137. doi: 10.17675/2305-6894-2019-8-4-19

17. A.H.M.J. Al-Obaidy, A. Kadhum, S.B. Al-Baghdadi, A. Al-Amiery, A.A.H. Kadhum, A.B. Mohamad and E. Yousif, Eco-friendly corrosion inhibitor: experimental studies on the corrosion inhibition performance of creatinine for mild steel in $\mathrm{HCl}$ complemented with quantum chemical calculations, Int. J. Electrochem. Sci., 2015, 10, 3961-3972.

18. A.A. Al-Amiery, A.A.H. Kadhum, A.B. Mohamad and S. Junaedi, A Novel Hydrazinecarbothioamide as a Potential Corrosion Inhibitor for Mild Steel in $\mathrm{HCl}$, Materials, 2013, 6, 1420-1431. doi: 10.3390/ma6041420

19. M.H.O. Ahmed, A.A. Al-Amiery, Y.K. Al-Majedy, A.A.H. Kadhum, A.B. Mohamad and T.S. Gaaz, Synthesis and characterization of a novel organic corrosion inhibitor for mild steel in $1 \mathrm{M}$ hydrochloric acid, Results Phys., 2018, 8, 728-733. doi: 10.1016/j.rinp.2017.12.039

20. T.A. Salman, A. Al-Amiery, L. Shaker, A. Kadhum, M. and Takriff. A study on the inhibition of mild steel corrosion in hydrochloric acid environment by 4-methyl-2(pyridin-3-yl)thiazole-5-carbohydrazide, Int. J. Corros. Scale Inhib., 2019, 8, no. 4, 1035-1059. doi: 10.17675/2305-6894-2019-8-4-14

21. E. Yousif, Y. Win, A. Al-Hamadani, A. Al-Amiery, A. Kadhum and A. Mohamad, Furosemide as an environmental-friendly inhibitor of corrosion of zinc metal in acid medium experimental and theoretical studies, Int. J. Electrochem. Sci., 2015, 10, $1708-$ 1718.

22. R.D. Salim, Q.A. Jawad, K.S. Ridah, L.M. Shaker, A.A. Al-Amiery, A.A.H. Kadhum and M.S. Takriff, Corrosion inhibition of thiadiazole derivative for mild steel in hydrochloric acid solution, Int. J. Corros. Scale Inhib., 2020, 9, no. 2, 550-561. doi: 10.17675/2305-6894-2020-9-2-10

23. A. Al-Amiery, L.M. Shaker, A.H. Kadhum and M.S. Takrif, Synthesis, characterization and gravimetric studies of novel triazole-based compound, Int. J. Low-Carbon Technol., 2020, 15, 164-170. 
24. E. Sheet, J. Vamin, H. Al-Salihi, A. Salam, K. Reda, W. Ahmed, M. Mahdi, A.A. AlAmiery, Corrosion N-(3-nitrobenzylidene)-2-aminobenzothiazole as new locally available corrosion inhibitor for Iraqi oil industry, J. Balk. Tribol. Assoc., 2020, 26, no. 2, 194-203.

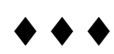

